\title{
Evaluation of TGF $\beta$, XPO4, elF5A2 and ANGPTL4 as biomarkers in HCC
}

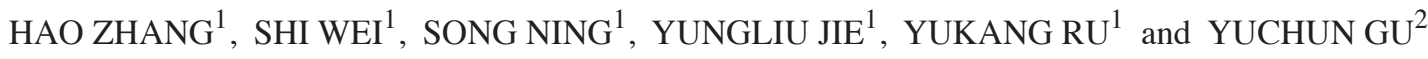 \\ ${ }^{1}$ Department of Surgery, Huashan Hospital, Fudan University, Shanghai 200040; \\ ${ }^{2}$ Institute of Molecular Medicine, Peking University, Beijing 100871, P.R. China
}

Received June 25, 2012; Accepted October 4, 2012

DOI: $10.3892 /$ etm.2012.750

\begin{abstract}
Hepatocellular carcinoma (HCC) is the most common type of liver cancer, and the fourth leading cause of cancer mortality worldwide. It is often diagnosed at an advanced stage, and hence typically has a poor prognosis. A number of distinct molecules have been recently identified as playing a role in the control of cancer progression. However, patients with HCC have a highly variable clinical course, indicating that $\mathrm{HCC}$ comprises several biologically distinctive subgroups reflecting a molecular heterogeneity of the tumors. To evaluate potential biomarkers in HCC, we employed multiple methods in this study, including qPCR, immunostaining methods and tissue microarrays (TMAs), as well as histological and pathological analysis, to assess TGF $\beta$, XPO4, elF5A2 and ANGPTL4 in cancerous and paracancerous liver tissues from 280 patients suffering from liver cancer. Our results found that all four indicators were located in the cytoplasm and distributed in cancerous and paracancerous liver tissues. Generally, there were higher levels of these indicators in paracancerous, compared with cancerous, liver tissues. These four indicators were correlated and modulated among each other. In connection with patient clinical and revisit information, statistical analysis determined that TGF $\beta 1$ in paracancerous liver tissue was positively correlated with tumor size. Higher production of TGF $\beta 1$ in paracancerous liver tissue was always associated with bigger liver tumors. $\mathrm{XPO} 4$ in cancerous liver tissue and TGF $\beta 1$ in paracancerous liver tissue were positively correlated with tumor differentiation. TGF $\beta 1$, ANGPTL 4 and elF5A2 were also positively correlated with the T classification of tumors. Additionally, higher levels of XPO4 in cancerous liver tissue suggested that the patient would have a better prognosis and survival rate. However, higher production of XPO4 in paracancerous liver tissue suggested a worse prognosis. All the results above
\end{abstract}

Correspondence to: Dr Hao Zhang, Department of Surgery, Huashan Hospital, Fudan University, Hua Shan Road, Shanghai 200040, P.R. China

E-mail: ycgu@pku.edu.cn

Key words: hepatocellular carcinoma, XPO4, TGF 31 , ANGPTL4, elF5A2, biomarker provide new insights into better understanding biological indicators, such as XPO4, TGF $\beta 1$, ANGPTL4 and elF5A2, in the prediction and evaluation of liver cancer, as well as signaling pathways in the control of liver cancer. XPO4 and TGF $\beta 1$ may serve as useful markers to evaluate the size and prognosis of liver cancer.

\section{Introduction}

Hepatocellular carcinoma (HCC) is one of the most common malignant tumors worldwide with an extremely high incidence and poor survival rate $(1,2)$. The management of patients at risk for developing $\mathrm{HCC}$ remains an intricate process. Despite the large number of studies devoted to the immunohistochemistry of $\mathrm{HCC}$, at the present time, the definitive positive and negative markers for HCC are lacking.

Several key molecules in signaling pathways involved in cancer development have emerged. TGF $\beta$ plays an important role in the regulation of cell growth and differentiation, angiogenesis, extracellular matrix formation, immunosuppression and cancer development (3). It is well known that signaling by the TGF $\beta$ family is most prominent at the interface between normal tissue development and cancer. The TGF $\beta$ signaling pathway is activated upon ligands binding to type I and II transmembrane receptors. The Smad4 protein is the downstream mediator of TGF $\beta$. Phosphorylation of Smad, by activation of TGF $\beta$ receptors, results in activation of a TGF $\beta$-targeted gene. ELF associates with SMAD3, presenting it to the cytoplasmic domain of the TGF $\beta$ receptor complex; this has been found to play a pivotal role in TGF $\beta$ signaling (4). Dysfunction of TGF $\beta$ pathway members, including TGF $\beta$ R2, SMAD3, SMAD4 and ELF, may lead to progenitor/stem cell deregulation and possibly cancer formation. Previous studies have suggested that Smad3 and its phosphorylation relatives may be used as biomarkers to identify patients with a high risk of recurrence $(5,6)$. Smad3 is exported via XPO4. XPO4 is therefore in control of Smad3 signaling as well as protein synthesis $(7,8)$. A recent study indicated that XPO4 may be involved in the progression of human HCC and may serve as a potential target for gene therapy in its treatment (9). We consequently selected $\mathrm{XPO} 4$ as an indicator in the current study.

HCC recurrence commonly occurs with an extremely poor prognosis. Vascular invasion in HCC is one of the key factors that results in cancer recurrence. To invade, HCC cells must 
penetrate the vessel wall, which consists of endothelial cells and extracellular matrix components, including fibronectin and fibrinogen. TGF $\beta$ specifically phosphorylates integrin $\beta 1$ via Smad-2 and Smad-3, causing a conformational change of the extracellular component with an inside-out mechanism (10). Additionally, a previous study in breast cancer revealed that the induction of ANGPTL4 by TGF $\beta$ via Smad disrupts vascular endothelial cell-cell junctions, increases the permeability of lung capillaries and facilitates the trans-endothelial passage of tumor cells (7,11-14).

Therefore, we employed multiple methods to assess TGF $\beta$, XPO4, elF5A2 and ANGPTL4 in cancerous and paracancerous liver tissue samples obtained from 280 patients suffering from liver cancer. We aimed to determine whether these four indicators may become biomarkers to evaluate HCC and provide an improved prognosis.

\section{Patients and methods}

Patients and samples. Samples were obtained under informed consent from 280 patients with HCC who underwent surgery to remove liver cancer between 2005 and 2011 in our hospital (Fudan University, Shanghai, China). All cases met the criteria set by the University of California, San Francisco (UCSF) (15). The follow-up of cases was at a mean of 42 months (range, 3-84 months). In patients with multi-nodular tumors, tumor samples were obtained from the largest tumor. Ethical approval was obtained from the Hua Shan Hospital Research Ethics Committee.

Tissue microarray (TMA) arrangement. TMA blocks were constructed as described previously (16). Briefly, all HCC tissues were reviewed by two histopathologists. Representative tumor areas free from necrotic and hemorrhagic materials were premarked in the paraffin blocks. Two cores, 1.5 or $2.0 \mathrm{~mm}$ in diameter, were taken from each representative tumor tissue, and from liver tissue adjacent to the tumor, and transferred from the recipient paraffin block at defined array positions. Six TMA blocks were constructed. Consecutive sections of $4-\mu \mathrm{m}$ thickness were taken on 3-aminopropyltriethoxysilane-coated slides (Shanghai Outdo Biotech Co., Ltd., Shanghai, China).

$q P C R$. RNA isolation and qRT-PCR was used. Total RNA was extracted from frozen tumor specimens and matched liver tissue adjacent to the tumor from 16 HCC patients using TRIzol reagent (Invitrogen Life Technologies, Carlsbad, CA, USA) according to the manufacturer's instructions. XPO4, TGF $\beta 1$, ANGPTL4 and elF5A2 mRNA expression in tissues from these patients was measured by qRT-PCR using an IQ5 instrument (Bio-Rad, Hercules, CA, USA). qRT-PCR was performed using a SYBR PrimeScript RT-PCR kit (Takara Bio, Inc., Shiga, Japan) according to the manufacturer's instructions. GAPDH was used as an internal control. The primers were as follows: XPO4 (Genbank NM_022459.4) forward primer 5'-TTGTTCTTGGTGTTTTGTGTTTCC-3' and reverse primer 5'-CATTCCTTTCCCACTCСТCTT TAG-3'; TGF $\beta 1$ (Genbank NM_000660.3) forward primer 5'-GCAACAATTCCTGGCGATACCT-3' and reverse primer 5'-CAGTGTGTTATCCCTGCTGTCACA-3'; ANGPTL4 (Genbank NM_001039667.1) forward primer 5'-GACCAA
GGGGCATGGAGCTT-3' and reverse primer 5'-CAGGGG ACCTACACACAACAGCA-3'; elF5A2 (Genbank NM_020390.5) forward primer 5'-TTGTTCTCAGGG CTATTTGTGCTAA-3' and reverse primer 5'-GGATGCTAC TGTTTCCATTTTTTTC-3'; and GAPDH (Genbank NM_002046.3) forward primer 5'-TCCCTCAACATTGTC AGCAA-3' and reverse primer 5'-AGCTCCACAACGGAT ACATT-3'. Relative mRNA levels were calculated based on the $\mathrm{Ct}$ values and corrected for GAPDH expression, according to the equation: $2^{-\Delta \mathrm{Ct}}[\Delta \mathrm{Ct}=\mathrm{Ct}$ (target gene) - $\mathrm{Ct}(\mathrm{GAPDH})]$. All experiments were performed in triplicate.

Immunostaining. The primary antibodies used for immunohistochemistry were XPO4 (polyclonal rabbit, diluted 1:100; PAB0297, Abnova, Taipei, Taiwan), TGF $\beta 1$ (monoclonal mouse, diluted 1:3,000; MAB2505, Abnova), ANGPTL4 (monoclonal mouse, diluted 1:200; L191591e, Enzo, NY, USA) and elF5A2 (polyclonal rabbit, diluted 1:30; E9781, Sigma, St. Louis, MO, USA). Immunohistochemistry was carried out using a two-step method as described previously (16). Following heat-induced antigen retrieval, tissues were incubated with primary antibodies for $30 \mathrm{~min}$ at room temperature. Following a 30-min incubation with a matched secondary antibody, sections were developed in 3,3'-diaminobenzidine solution (Sigma) under microscopic observation and counterstained with hematoxylin (Sigma). Negative control slides in which the primary antibodies had been omitted were included in all assays.

Statistical analysis. Analysis was performed with SPSS 17.0 for Windows (SPSS Inc., Chicago, IL, USA); the values are expressed as the mean \pm standard error. A two-tailed P-value of $<0.05$ was considered to indicate a statistically significant difference. The differential expression of proteins between carcinoma tissue and adjacent tissue was determined by a t-test. In statistics, correlation refers to any of a broad class of statistical relationships involving dependence. The most common method is the Pearson correlation coefficient (CC), which is sensitive only to a linear relationship between two variables. Two variables are more correlative if the $\mathrm{CC}$ value is closer to 1. Relationships between clinicopathological and molecular parameters were statistically analyzed using Pearson or Spearman's rank correlation coefficients. $\mathrm{P}<0.05$ indicates that the two groups are correlative. Kaplan-Meier analysis was used to determine survival. The log-rank test was then used to compare patient survival between subgroups.

\section{Results}

Distribution of indicators in liver tissues. The four indicators, XPO4, TGF 31 , ANGPTL4 and elF5A2, were found to be located within the cellular cytoplasm by the immunostaining method (Fig. 1). The data shown in Table I are based on the immunohistochemical staining levels. The values in the table were obtained from the percentage of positive staining in the same samples among TMAs. With regard to tissue distribution of the 4 indicators, they were all expressed in cancerous and paracancerous liver tissues. They were all found at a significantly higher density in the paracancerous tissues than in the cancerous liver tissues (Table I). 
Table I. Expression of XPO4, TGF $\beta 1$, ANGPTL4 and elF5A2.

\begin{tabular}{lccr}
\hline Indicator & Carcinoma tissue & Adjacent tissue & P-value $^{\mathrm{a}}$ \\
\hline XPO4 & $0.800 \pm 0.194$ & $0.855 \pm 0.113$ & 0.000 \\
TGFß1 & $0.256 \pm 0.284$ & $0.502 \pm 0.312$ & 0.000 \\
ANGPTL4 & $0.723 \pm 0.247$ & $0.817 \pm 0.173$ & 0.000 \\
elF5A2 & $0.770 \pm 0.176$ & $0.814 \pm 0.141$ & 0.000
\end{tabular}

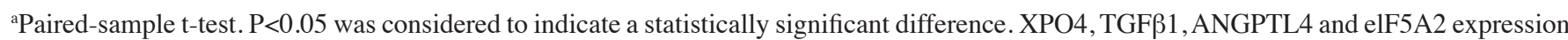
in carcinoma tissues was significantly lower than that in adjacent tissues.

Correlation in expression among these indicators. The correlation in expression of XPO4 between the cancerous and paracancerous liver tissue was positive $(\mathrm{CC}=0.304$, $\mathrm{P}<0.001)$. Expression of XPO4 in the cancerous liver tissue was positively correlated with expression of TGF $\beta 1$ $(\mathrm{CC}=0.126, \mathrm{P}=0.047)$ in paracancerous liver tissue, expression of ANGPTL4 $(\mathrm{CC}=0.506, \mathrm{P}=0.000)$ in cancerous liver tissue, expression of ANGPTL4 $(\mathrm{CC}=0.199, \mathrm{P}=0.002)$ in paracancerous liver tissue and expression of elF5A2 $(C C=0.194$, $\mathrm{P}=0.002$ ) in paracancerous liver tissue (Table II). The correlation in expression of ANGPTL4 between the cancerous and paracancerous liver tissue was positive $(\mathrm{CC}=0.282, \mathrm{P}<0.001)$. Expression of ANGPTL4 in the cancerous liver tissue was positively correlated with expression of XPO4 $(\mathrm{CC}=0.506$, $\mathrm{P}<0.001)$ in carcinoma liver tissue, expression of elF5A2 $(\mathrm{CC}=0.469, \mathrm{P}<0.001)$ in carcinoma liver tissue and expression of elF5A2 $(\mathrm{CC}=0.245, \mathrm{P}<0.001)$ in paracancerous liver tissue. The correlation in expression of elF5A2 between cancerous and paracancerous liver tissues was positive $(\mathrm{CC}=0.371$, $\mathrm{P}<0.001)$. Expression of elF5A2 in the cancerous liver tissue was positively correlated with expression of XPO4 $(C C=0.478$, $\mathrm{P}<0.001)$ in carcinoma liver tissue. These results suggest that the expression of these four indicators is internally connected and there is modulation between each of them.

Correlation between expression of indicators and pathological information

Indicators and tumor size. In patients with multi-nodular tumors, the tumor samples were obtained from the largest tumor. The statistical results revealed that the expression of TGF $\beta 1$ in paracancerous liver tissue was significantly positively correlated with tumor size $(C C=0.147, P=0.021, n=248$; Table III). The other 7 parameters, e.g., TGF $\beta 1$ in cancerous liver tissue, and XPO4 in cancerous liver tissue, had no significant correlation with tumor size.

Indicators and blood vessel invasion The statistical results revealed that all indicators in cancerous and paracancerous liver tissues had no significant correlation with blood vessel invasion (Table IV).

Indicators and pathological classification (differentiation). The patients were divided into two categories according to the Edmondson classification; high differentiation (I, II, I-II) and low differentiation (II-III, III, IV). The statistical results revealed that all indicators exhibited higher expression levels in the low differentiation group than in the high differentiation group (Table V). XPO4 in cancerous liver tissue $(\mathrm{CC}=0.143, \mathrm{P}=0.035)$ and $\mathrm{TGF} \beta 1(\mathrm{CC}=0.195, \mathrm{P}=0.004)$ in paracancerous liver tissue were significantly correlated with tumor differentiation.

Indicators and tumor $T$ classification The statistical results revealed that expression of TGF $\beta 1$ in both cancerous and paracancerous tissues $(\mathrm{CC}=0.402, \mathrm{P}=0.000 ; \mathrm{CC}=0.299$, $\mathrm{P}=0.000$, respectively) was positively correlated with $\mathrm{T}$ classification; expression of ANGPTL4 in cancerous and paracancerous liver tissues $(\mathrm{CC}=0.125, \mathrm{P}=0.049 ; \mathrm{CC}=0.142$, $\mathrm{P}=0.025$, respectively) was positively correlated with $\mathrm{T}$ classification and that the expression of elF5A2 in paracancerous liver tissues $(\mathrm{CC}=0.127, \mathrm{P}=0.047)$ was positively correlated with $\mathrm{T}$ classification.

Indicators and survival function. Kaplan-Meier analysis indicated that the expression of XPO4 in carcinoma tissue did not correlate with survival function in overexpression and underexpression $(\mathrm{P}=0.202)$. The survival plot indicated that survival rates in patients with XPO4 overexpression were higher than those in patients with XPO4 underexpression (Fig. 2A). Expression of XPO4 in adjacent tissue did not correlate with overexpression or underexpression $(\mathrm{P}=0.139)$. The survival plot indicated that survival rates in patients with XPO4 overexpression in adjacent tissues were lower than those in patients with XPO4 underexpression. These results suggested that higher expression of XPO4 in cancerous liver tissue was indicative that the patient would have a better prognosis and increased survival rate. However, higher concentrations of XPO4 in paracancerous liver tissue suggested a worse prognosis (Fig. 2B). Furthermore, expression of TGF $\beta 1$ in carcinoma tissue did not correlate with overexpression or underexpression $(\mathrm{P}=0.954)$. The survival figure indicated that patients who were positive for TGF $\beta 1$ in cancerous liver tissue had a better prognosis than those who were negative for TGF $\beta 1$ in cancerous liver tissue (Fig. 3). Other factors, e.g., ANGPTL4 and ELF, were not correlated with overexpression or underexpression in either of the cancerous and adjacent tissues.

\section{Discussion}

In the present study, we employed multiple techniques, including the use of qPCR, immunostaining and TMAs, as well as histology and pathology analysis, to undertake a study to evaluate XPO4, TGF $\beta 1$, ANGPTL4 and elF5A2 in carcinoma and paracarcinoma tissues from 280 liver cancer patients. Our results revealed that all four indicators were 


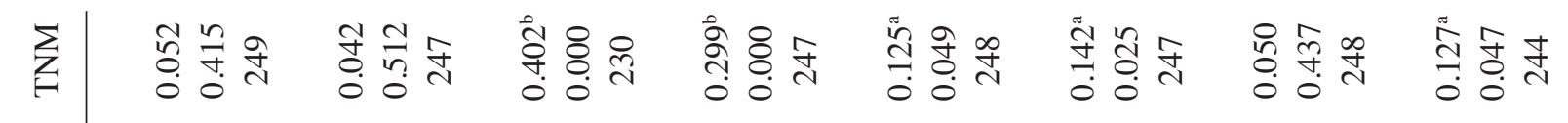

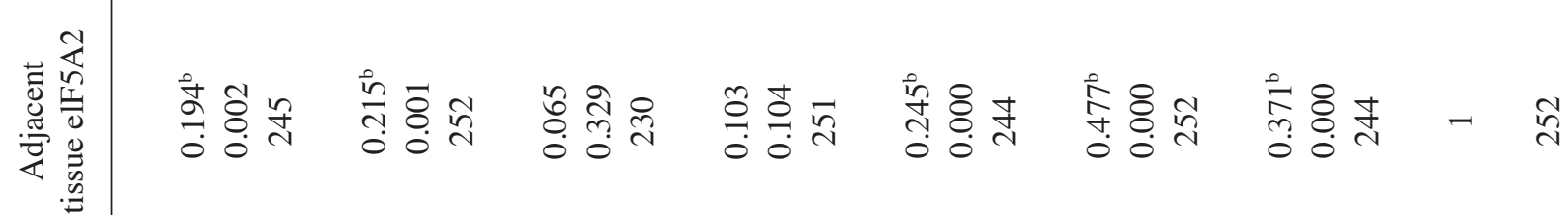

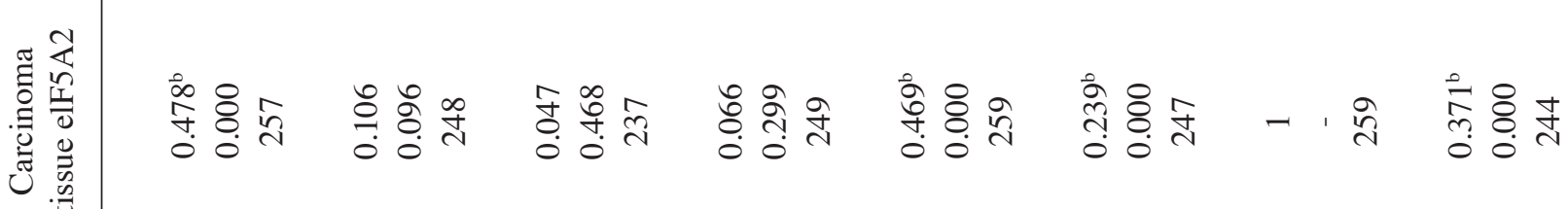

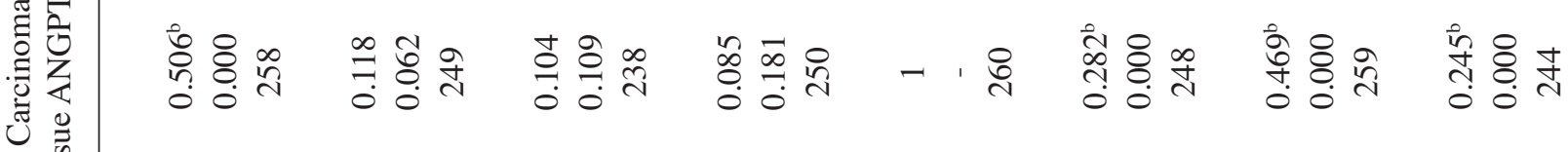

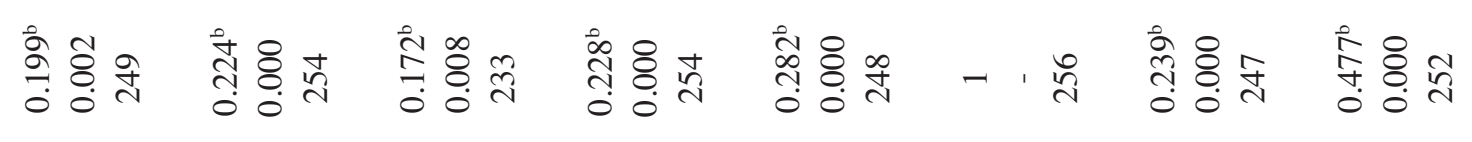

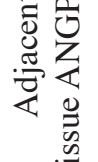

$\stackrel{+}{\exists}$

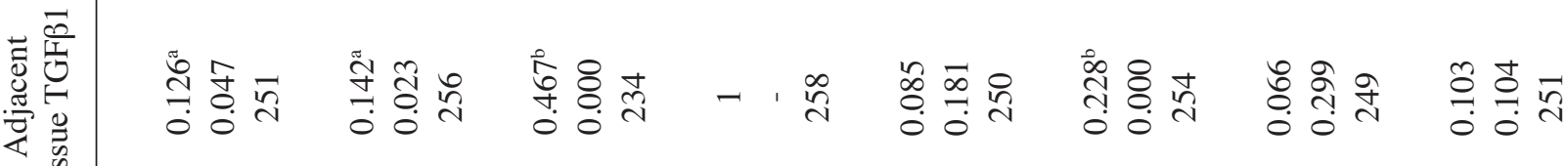

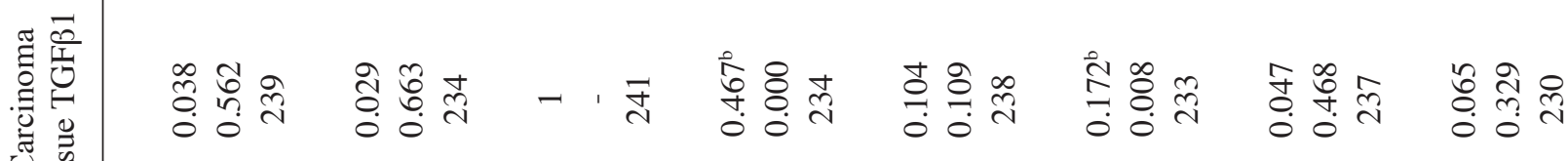

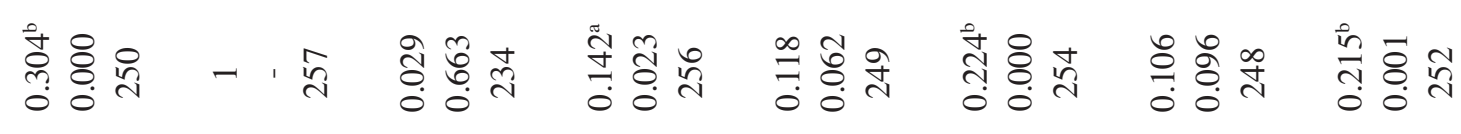

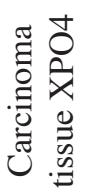

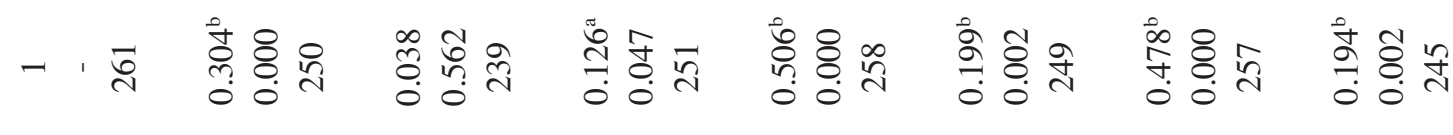

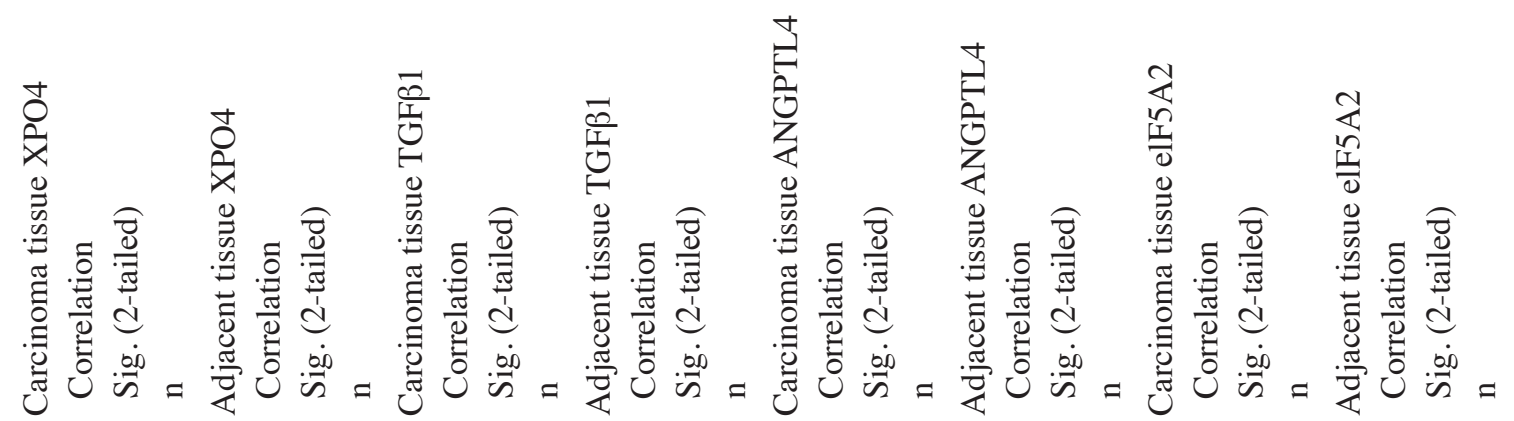



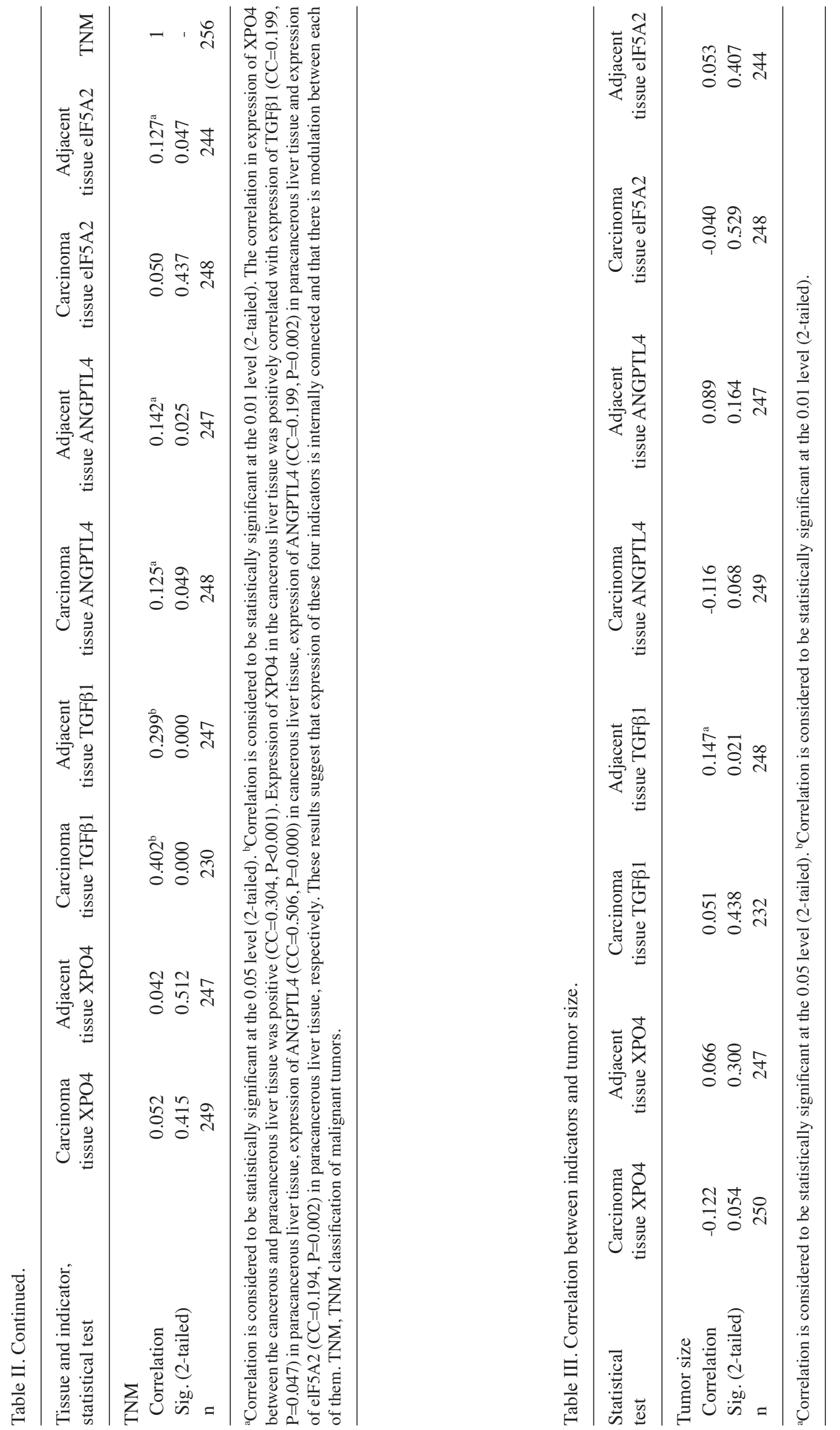
Table IV. Correlation between XPO4, TGF $\beta 1$, ANGPTL4 and elF5A2 expression and vascular invasion in carcinoma tissue and adjacent tissue.

\begin{tabular}{|c|c|c|c|c|}
\hline Indicator & Tissue type & Vascular invasion (yes) & Vascular invasion (no) & P-value \\
\hline \multirow[t]{2}{*}{ XPO4 } & Carcinoma tissue & $0.689 \pm 0.317$ & $0.803 \pm 0.185$ & 0.313 \\
\hline & Adjacent tissue & $0.806 \pm 0.174$ & $0.855 \pm 0.111$ & 0.417 \\
\hline \multirow[t]{2}{*}{ TGF $\beta 1$} & Carcinoma tissue & $0.259 \pm 0.300$ & $0.257 \pm 0.281$ & 0.984 \\
\hline & Adjacent tissue & $0.539 \pm 0.227$ & $0.502 \pm 0.311$ & 0.724 \\
\hline \multirow[t]{2}{*}{ ANGPTL4 } & Carcinoma tissue & $0.780 \pm 0.132$ & $0.723 \pm 0.247$ & 0.465 \\
\hline & Adjacent tissue & $0.889 \pm 0.042$ & $0.816 \pm 0.172$ & 0.210 \\
\hline \multirow[t]{2}{*}{ elF5A2 } & Carcinoma tissue & $0.760 \pm 0.145$ & $0.769 \pm 0.174$ & 0.861 \\
\hline & Adjacent tissue & $0.867 \pm 0.070$ & $0.812 \pm 0.143$ & 0.258 \\
\hline
\end{tabular}

The statistical results revealed that all indicators in cancerous and paracancerous liver tissue had no significant correlation with blood vessel invasion.

Table V. Association of XPO4, TGF $\beta 1$, ANGPTL4 and elF5A2 expression with differentiation.

\begin{tabular}{|c|c|c|c|c|}
\hline Indicator & Tissue type & High differentiation & Low differentiation & P-value \\
\hline \multirow[t]{2}{*}{ XPO4 } & Carcinoma tissue & $0.793 \pm 0.195$ & $0.850 \pm 0.150$ & 0.035 \\
\hline & Adjacent tissue & $0.849 \pm 0.115$ & $0.881 \pm 0.106$ & 0.054 \\
\hline \multirow[t]{2}{*}{ TGF $\beta 1$} & Carcinoma tissue & $0.312 \pm 0.290$ & $0.303 \pm 0.269$ & 0.867 \\
\hline & Adjacent tissue & $0.540 \pm 0.285$ & $0.658 \pm 0.245$ & 0.003 \\
\hline \multirow[t]{2}{*}{ ANGPTL4 } & Carcinoma tissue & $0.751 \pm 0.213$ & $0.737 \pm 0.211$ & 0.643 \\
\hline & Adjacent tissue & $0.833 \pm 0.148$ & $0.842 \pm 0.121$ & 0.689 \\
\hline \multirow[t]{2}{*}{ elF5A2 } & Carcinoma tissue & $0.775 \pm 0.170$ & $0.800 \pm 0.106$ & 0.253 \\
\hline & Adjacent tissue & $0.816 \pm 0.152$ & $0.845 \pm 0.082$ & 0.080 \\
\hline
\end{tabular}

Correlation regression analysis indicated that expression of TGF $\beta 1$ in adjacent tissue and XPO4 in carcinoma tissue were significantly correlated with differentiation. $\mathrm{XPO} 4$ of carcinoma tissue, $\mathrm{CC}=0.143, \mathrm{P}=0.035$; TGF $\beta 1$ of adjacent tissue, $\mathrm{CC}=0.195, \mathrm{P}=0.004$. $\mathrm{CC}$, correlation coefficient .

located in the cytoplasm and distributed in both cancerous and paracancerous liver tissues. Generally, there were higher levels of these indicators in paracancerous than in cancerous liver tissue. The correlation analysis results further revealed that expression levels of these four indicators were correlated and modulated amongst each other. In connection with patients' clinical and revisit information, statistical results revealed that TGF $\beta 1$ levels in paracancerous liver tissue was positively correlated with the tumor size. Higher levels of TGF $\beta 1$ in paracancerous liver tissue were always associated with bigger liver tumors. XPO4 in cancerous liver tissue and TGF $\beta 1$ in paracancerous liver tissue were positively correlated with tumor differentiation. TGF $\beta 1$, ANGPTL4 and elF5A2 were also positively correlated with the $\mathrm{T}$ classification of tumors. Additionally, a higher expression of XPO4 in cancerous liver tissue suggested that the patient would have a better prognosis and survival rate. However, higher XPO4 levels in paracancerous liver tissue suggested a worse prognosis. These results suggest that XPO4 may be a potential biomarker to assess HCC prognosis and differentiation.

In the present study, we performed experimental and statistical work in cancerous and paracancerous materials from 280 patients suffering from liver cancer. All patient materials were collected and preserved according to standard protocols (see Patients and methods). In order to reduce the experimental error/variance obtained by processing different tissue samples using immunostaining, we selected the method of TMAs to equalize the experimental condition among different samples. TMA is an innovative type of specimen slide with multiple samples on a single slide, which was first described in $1986(17,18)$. In our study, each slide integrated 200 tissue specimens on a single slide as 500 dots, which represented multiple tissues, pathological types, patients and stages, as well as control samples, aiding in the creation of a low margin for error. This method allows for high throughput of data of multiple patient cases and control samples simultaneously. Thus reagent concentrations were identical for each case, as were incubation times, temperatures and wash conditions. This greatly improves the accuracy of the results, leading to more confident conclusions (19). Each experiment was also repeated in three slides of TMAs. Data averages were used for analysis.

We have been aware of the result complicity from different patients and made numerous attempts to summarize our 
A

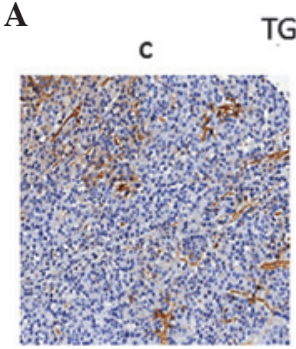

C

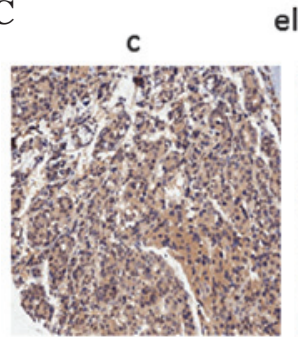

TGF $\beta$

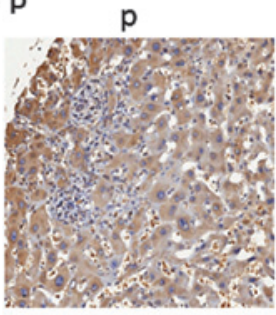

elF

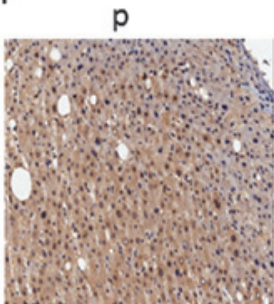

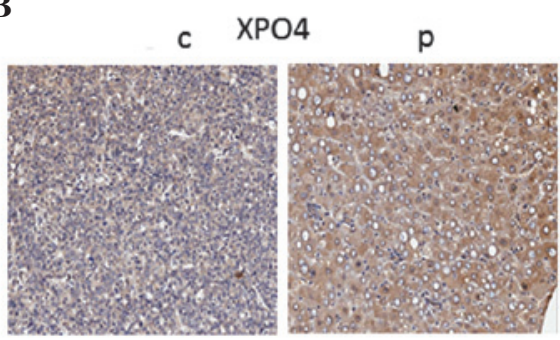

D

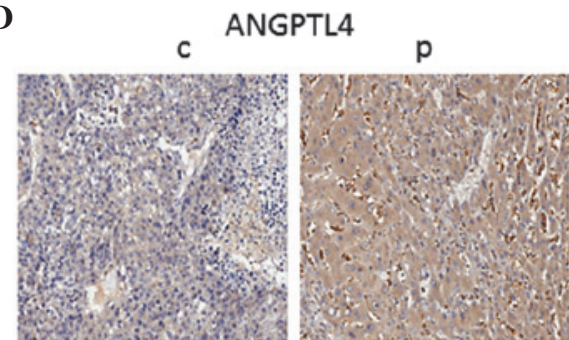

Figure 1. Immunostaining images for TGF $31, \mathrm{XPO} 4, \mathrm{ANGPTL} 4$ and elF5A2 in carcinoma and paracarcinoma tissues. $\mathrm{c}$ indicates the carcinoma tissue and $\mathrm{p}$ indicates the paracarcinoma tissue.

A

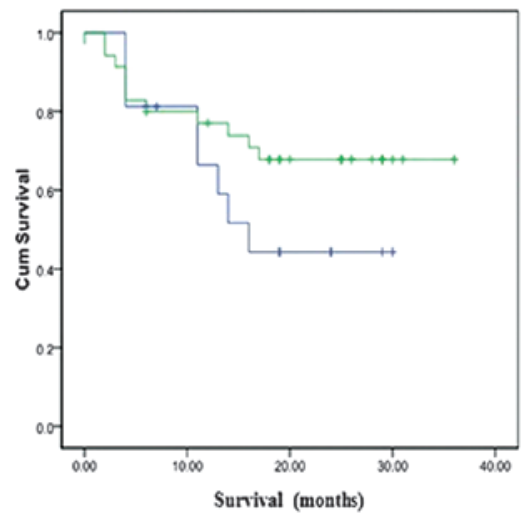

B

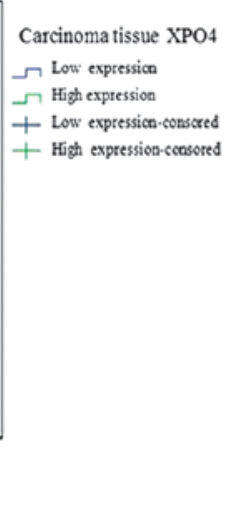

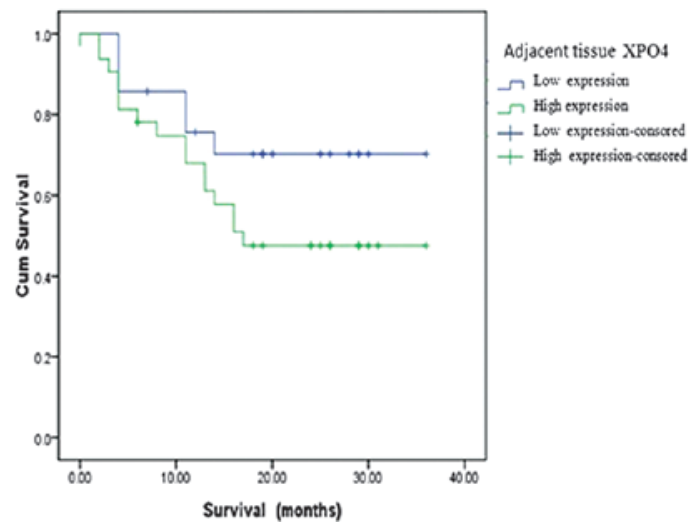

Figure 2. Kaplan-Meier analysis of XPO4 correlated with patient survival function. (A) Kaplan-Meier analysis indicated that expression of XPO4 in carcinoma tissue did not correlate with overexpression or underexpression $(\mathrm{P}=0.202)$, and that survival rates of XPO4-overexpressing patients were higher than those of patients with XPO4 underexpression. (B) Kaplan-Meier analysis indicated that expression of XPO4 in adjacent tissue did not correlate with overexpression or underexpression $(\mathrm{P}=0.139)$, and that survival rates of XPO4-overexpressing patients were lower than those of patients with XPO4 underexpression.

A

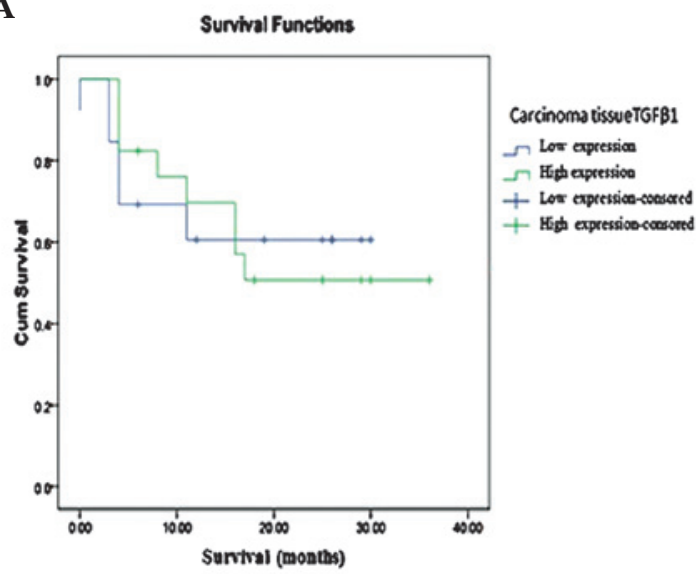

B Sunvival functions

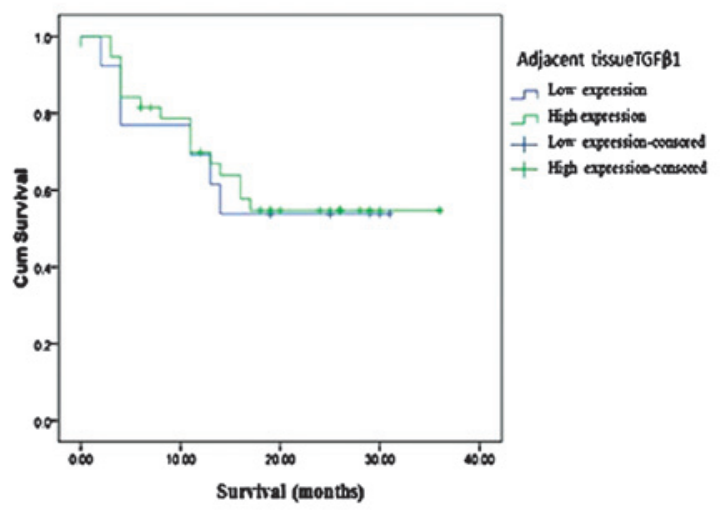

Figure 3. Kaplan-Meier analysis of TGF $\beta 1$ in adjacent tissue correlated with patient survival. Expression of TGF $\beta 1$ in carcinoma tissue (A) was not correlated with overexpression and underexpression $(\mathrm{P}=0.954)$. The results indicated that expression of TGF $\beta 1$ in adjacent tissue (B) was not correlated with overexpression and underexpression $(\mathrm{P}=0.884)$. 
results in a realistic way. It is conventional to take the middle point as a standard to perform analysis in survival function. However, there is no meaning because the positive probability of indicators displays skew distribution. The 25 th percentile was therefore eventually taken for correlation analysis in our study. The suggestion given by this analysis is rational and it is close to clinic observation by current knowledge.

TGF $\beta$ is a multipotent polypeptide which regulates cell proliferation, differentiation and apoptosis and deters tumor growth (20). Within the tumor micro-environment, TGF $\beta$ is produced by liver cancer cells. It is located within the cytoplasm, as shown in our results and consistently in a number of previous studies $(3,5)$. However, as a natural response to the hypoxic and inflammatory conditions that occur during tumor progression, high production levels of TGF $\beta 1$ in our study were unexpectedly found in paracancerous, but not cancerous, liver tissue. Moreover, TGF $\beta 1$ in cancerous or paracancerous liver tissue had no connection with either tumor vessel invasion or lymph metastasis. These results were inconsistent with previous results which demonstrated that the overexpression of TGF $\beta 1$ is associated with a high incidence of distant metastasis and that TGF $\beta 1$ promotes vascular invasion by activating $\beta 1$ integrin $(10,21)$. Additionally, patients positive for TGF $\beta 1$ in the cancerous tissue were suggested to have a better prognosis, as is consistent with a previous report (6). This result may partly support the current concept for a dual role of the TGF $\beta$ signaling pathway in hepatocellular cancer suppression and progression of differentiation $(3,6,22,23)$.

TGF $\beta$ receptors phosphorylate $\mathrm{Smad} 3$ and induce its nuclear import, then regulate gene transcription. Smad3 returns to the cytoplasm to propagate further cycles of signal transduction. In HCCs, Smad3 and its phosphorylation relatives have been suggested to be the predictors of prognosis in patients with liver cancer and also serve as the biomarkers to identify patients with a high risk of recurrence (5). Smad3 is exported via XPO4. XPO4 is therefore in control of Smad3 signaling as well as protein synthesis $(7,8)$. Consistent with a previous study (9), our results also demonstrated that XPO4 was present in cancerous and paracancerous liver tissues, with a higher density of XPO4 in paracancerous tissue. Our results found that TGF $\beta 1$, but not XPO4 (9), in paracancerous tissue was significantly positively correlated with tumor size and histopathological classification. Moreover, our results also suggested that high XPO4 in cancerous tissue resulted in a good prognosis. This is consistent with a previous study which indicated that downregulation of XPO4 resulted in a poor prognosis (9). Our results in an adequate sample size emphasized again that XPO4 may be involved in the progression of human HCC and may serve as a potential biomarker to evaluate the condition. A further validation study is suggested on a bigger sample size. Additionally, assessment of tumor behavior in HCC cell lines with or without rescuing XPO4 may confirm the therapeutic role of XPO4 in HCC.

elF5A2 is amplified in human tumors, is required for proliferation of XPO4-deficient tumor cells and promotes HCC in mice (7). Our results provided evidence of correlation among these four indicators in HCC. Production of elF5A2 in paracancerous tissue is significantly positively associated with cancer histopathological classification. Previous studies have also revealed that the induction of angiopoietin-like 4 (ANGPTL4) by TGF $\beta$, via the Smad signaling pathway, is critical for the trans-endothelial passage of tumor cells resulting in tumor metastasis (14). Further studies have revealed that ANGPTL4 regulates endothelial cell junction organization (24) and pericyte coverage (11), resulting in disruption in endothelial cell-cell junctions (25). We therefore carried out the rational measurement of ANGPTL4 in the cancerous and paracancerous liver tissues in a variety of patients. Our results showed that there was no significant correlation between ANGPTL4 and vessel or lymphatic invasion. It is inconsistent with previous reports that expression of ANGPTL4 was statistically correlated with the degree of differentiation, lymphatic invasion and venous invasion $(12,26)$. Our results provided evidence that ANGPTL4 is not a metastasis-inducing factor in HCC.

In summary, the results of the present study revealed that XPO4, TGF 31 , ANGPTL4 and elF5A2 were present in both cancerous and paracancerous liver tissues, and that they were closely correlated with each other. TGF $\beta 1$ in paracancerous liver tissue was positively correlated with the tumor size. XPO4 in cancerous liver tissue and TGF $\beta 1$ in paracancerous liver tissue were positively associated with tumor differentiation. Meanwhile, TGF $\beta 1$, ANGPTL4 and elF5A2 were significantly correlated with the $\mathrm{T}$ classification of tumors. Of these four indicators, XPO4 appears to be a potential biomarker to evaluate HCC.

\section{Acknowledgements}

This study was supported by a grant from Scientific Research Projects of Health Bureau of Shanghai (09411952800) to HZ. This study was approved by the Hua Shan Hospital, Fudan University Administration Panel of Clinic research, China.

\section{References}

1. Parkin DM: Global cancer statistics in the year 2000. Lancet Oncol 2: 533-543, 2001

2. Fabregat I: Dysregulation of apoptosis in hepatocellular carcinoma cells. World J Gastroenterol 15: 513-520, 2009.

3. Dong ZZ, Yao DF, Yao M, et al: Clinical impact of plasma TGF-beta1 and circulating TGF-beta1 mRNA in diagnosis of hepatocellular carcinoma. Hepatobiliary Pancreat Dis Int 7: 288-295, 2008

4. Mishra L, Derynck R and Mishra B: Transforming growth factorbeta signaling in stem cells and cancer. Science 310: 68-71, 2005.

5. Kim SH, Ahn S and Park CK: Smad3 and its phosphoisoforms are prognostic predictors of hepatocellular carcinoma after curative hepatectomy. Hepatobiliary Pancreat Dis Int 11: 51-59, 2012.

6. Coulouarn C, Factor VM and Thorgeirsson SS: Transforming growth factor-beta gene expression signature in mouse hepatocytes predicts clinical outcome in human cancer. Hepatology 47: 2059-2067, 2008.

7. Zender L, Xue W, Zuber J, et al: An oncogenomics-based in vivo RNAi screen identifies tumor suppressors in liver cancer. Cell 135: 852-864, 2008.

8. Kurisaki A, Kurisaki K, Kowanetz M, et al: The mechanism of nuclear export of Smad3 involves exportin 4 and Ran. Mol Cell Biol 26: 1318-1332, 2006.

9. Liang XT, Pan K, Chen MS, et al: Decreased expression of XPO4 is associated with poor prognosis in hepatocellular carcinoma. J Gastroenterol Hepatol 26: 544-549, 2011.

10. Fransvea E, Mazzocca A, Antonaci S and Giannelli G: Targeting transforming growth factor (TGF)-betaRI inhibits activation of betal integrin and blocks vascular invasion in hepatocellular carcinoma. Hepatology 49: 839-850, 2009.

11. Perdiguero EG, Galaup A, Durand M, et al: Alteration of developmental and pathological retinal angiogenesis in angpt14-deficient mice. J Biol Chem 286: 36841-36851, 2011. 
12. Li H, Ge C, Zhao F, et al: Hypoxia-inducible factor alpha-activated angiopoietin-like protein 4 contributes to tumor metastasis via vascular cell adhesion molecule-1/integrin $\beta 1$ signaling in human hepatocellular carcinoma. Hepatology 54: 910-919, 2011.

13. Huang RL, Teo Z, Chong HC, et al: ANGPTL4 modulates vascular junction integrity by integrin signaling and disruption of intercellular VE-cadherin and claudin-5 clusters. Blood 118 : 3990-4002, 2011.

14. Padua D, Zhang XH, Wang Q, et al: TGFbeta primes breast tumors for lung metastasis seeding through angiopoietin-like 4 . Cell 133: 66-77, 2008

15. Yao FY, Ferrell L, Bass NM, et al: Liver transplantation for hepatocellular carcinoma: expansion of the tumor size limits does not adversely impact survival. Hepatology 33: 1394-1403, 2001

16. Gao Q, Qiu SJ, Fan J, et al: Intratumoral balance of regulatory and cytotoxic $\mathrm{T}$ cells is associated with prognosis of hepatocellular carcinoma after resection. J Clin Oncol 25: 2586-2593, 2007.

17. Battifora H: The multitumor (sausage) tissue block: novel method for immunohistochemical antibody testing. Lab Invest 55: 244-248, 1986.

18. Kononen J, Bubendorf L, Kallioniemi A, et al: Tissue microarrays for high-throughput molecular profiling of tumor specimens. Nat Med 4: 844-847, 1998.

19. Xiang ZL, Zeng ZC, Tang ZY, et al: Potential prognostic biomarkers for bone metastasis from hepatocellular carcinoma. Oncologist 16: 1028-1039, 2011.
20. Giannelli G, Mazzocca A, Fransvea E, Lahn M and Antonaci S: Inhibiting TGF $\beta$ signaling in hepatocellular carcinoma. Biochim Biophys Acta 1815: 214-223, 2011.

21. Picon A, Gold LI, Wang J, Cohen A and Friedman E: A subset of metastatic human colon cancers expresses elevated levels of transforming growth factor beta1. Cancer Epidemiol Biomarkers Prev 7: 497-504, 1998.

22. Mishra L, Banker T, Murray J, et al: Liver stem cells and hepatocellular carcinoma. Hepatology 49: 318-329, 2009.

23. Baek HJ, Lim SC, Kitisin K, et al: Hepatocellular cancer arises from loss of transforming growth factor beta signaling adaptor protein embryonic liver fodrin through abnormal angiogenesis. Hepatology 48: 1128-1137, 2008.

24. Cazes A, Galaup A, Chomel C, et al: Extracellular matrix-bound angiopoietin-like 4 inhibits endothelial cell adhesion, migration, and sprouting and alters actin cytoskeleton. Circ Res 99: 1207-1215, 2006

25. Hasita H, Komohara Y, Okabe H, et al: Significance of alternatively activated macrophages in patients with intrahepatic cholangiocarcinoma. Cancer Sci 101: 1913-1919, 2010.

26. Shibata K, Nakayama T, Hirakawa H, Hidaka S and Nagayasu T: Clinicopathological significance of angiopoietin-like protein 4 expression in oesophageal squamous cell carcinoma. J Clin Pathol 63: 1054-1058,2010. 